\title{
A Solution for Estimating the Tensile Yield Strength From Small Specimens
}

REFERENCE: Mak, Jonathan, Wei, Tao, Wuhrer, Richard, Yeung, Wing, Heness, Greg, and Di, Zhang, "A Solution for Estimating the Tensile Yield Strength From Small Specimens," Journal of Testing and Evaluation, Vol. 41, No. 4, 2013, pp. 1-4, doi:10.1520/JTE20120230. ISSN 0090-3973.

\begin{abstract}
The small punch test is an innovative test that utilises small disc-shaped specimens to assess the mechanical behaviour of materials. The main advantage is the relatively small specimen size. In this article, a modified analytical solution for the small punch maximum bend strength is proposed that is based on classical plate theory. A clear linear relationship is observed between the tensile yield strength $\sigma_{\mathrm{YS}}$ and the small punch maximum bend strength $\sigma_{y}$ for both alloys and metal matrix composites.
\end{abstract}

KEYWORDS: small punch test, metal matrix composite, yield strength, equivalent contact radius

\section{Introduction}

Studies have been conducted regarding prediction of the tensile yield strength $\sigma_{\mathrm{YS}}$ from small punch load-displacement curves. Mao and Takahashi proposed an empirical relation of the small punch elastic-plastic load $P_{y}$ and the tensile yield strength $\sigma_{\mathrm{YS}}$ for ferritic steels as shown in Eq 1 [1], where $P_{y}$ is the small punch elastic-plastic load and $t_{0}$ is the original thickness. The theory is based on the assumption that the strength of a material is proportional to the load via an empirical relationship. Furthermore, the small punch elastic-plastic load $P_{y}$ is described by many authors as representing the end of elastic bending and the beginning of localised plastic deformation [2].

$$
\sigma_{y}=0.360 \times \frac{P_{y}}{t_{0}^{2}}
$$

This assumption was further analysed by $\mathrm{Xu}$ and Zhao, who developed an analytical solution based on analysis of the elasticplastic bulge deformation behaviour of a circular plate and for very small deflections as described in Eq 2 [3].

$$
\sigma_{y}=\frac{3}{2 \pi} \times \frac{P_{y}}{t_{0}^{2}}
$$

Cheon and Kim described this relationship between the tensile yield strength $\sigma_{\mathrm{YS}}$ and the small punch elastic-plastic load $\sigma_{y}$

Manuscript received July 13, 2012; accepted for publication January 22, 2013; published online May 24, 2013.

${ }^{1}$ Dept. of Physics and Advanced Materials, Univ. of Technology, Sydney, Ultimo, NSW 2007, Australia.

${ }^{2}$ Institute of Materials Engineering, Australian Nuclear Science and Technology Organisation, Lucas Heights, NSW 2234, Australia.

${ }^{3}$ Advanced Materials Characterisation Facility, Univ. of Western Sydney, Penrith, NSW 2751, Australia.

${ }^{4}$ State Key Laboratory of Metal Matrix Composites, Shanghai Jiao Tong Univ., Shanghai 200030. using a normalisation parameter, $\alpha$ [4]. Furthermore, Finarelli et al. claimed that $\alpha$ is influenced by material properties and test geometry [5] as expressed in Eq 3.

$$
\sigma_{y}=\alpha \times \frac{P_{y}}{t_{0}^{2}}
$$

Eskner and Sandström analysed the initial elastic deformation using classical plate bend theory as shown in Eq 4 [6], where again $P_{y}$ is the small punch elastic-plastic load, $t_{0}$ is the original thickness, $R_{1}$ is the radius of the lower die bore, $v$ is Poisson's ratio, and $r^{\prime}$ is the small punch equivalent contact radius. In their work, uniaxial and biaxial flow properties are found to be in good agreement for $1 \mathrm{Cr}-0.5 \mathrm{Mo}$ low alloy and $18 \mathrm{Cr}-9 \mathrm{Ni}$ steels. The solution takes into account the geometry of the spherical punch contact radius with respect to the small punch configuration for the clamped condition. Note that this analysis assumes primarily elastic bending, for which the effects imparted by punch indentation are assumed to be negligible [2].

$$
\sigma_{y}=\frac{3 P_{y}(1+\nu)}{2 \pi t_{0}^{2}} \ln \frac{R_{1}}{r^{\prime}}
$$

The main problem in this interpretation is the characterisation of the equivalent contact radius $r^{\prime}$, which has been proposed by a number of authors as shown in Table 1.

The current view on the equivalent contact radius $r^{\prime}$ is based on Westergaard's solution [10]; however, Fleury and Ha provide a

TABLE 1—Proposed small punch equivalent contact radius $r^{\prime}$.

\begin{tabular}{lcc}
\hline Author & $r^{\prime}$ & Constraint \\
\hline Westergard [7] & $\sqrt{1.6 r_{o}^{2}+t_{0}^{2}}-0.675 t_{0}$ & $0 \leq r_{o} \leq \frac{t_{0}}{2}$ \\
Fleury and Ha [8] & $0.721(P d \gamma)^{1 / 3}$ & $r_{o} \geq 0$ \\
Shetty [9] & $\frac{t_{0}}{3}$ & $r_{o} \geq 0$ \\
\hline
\end{tabular}




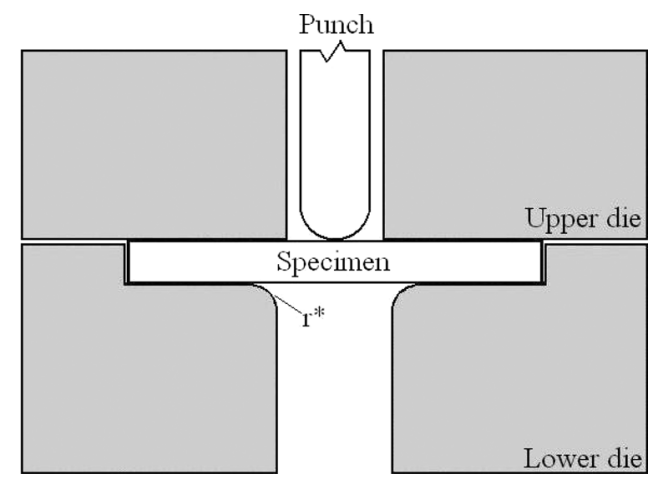

FIG. 1-Schematic representation of the small punch test.

better solution, as it acknowledges the influence of Young's modulus $E$ and Poisson's ratio $v$ [8] for both the spherical punch and the specimen. Nevertheless, the solution requires prior knowledge of the material's mechanical properties, which are often not readily available.

Another approach for determining the yield strength from a small punch test (SPT) is based on considering the plasticity of the material. This approach attempts to overcome the potential problems of determining yield strength simply from the linearity deviation. A number of works involving interpretations that include full consideration of plasticity from initial loading through to failure are found in the literature. This includes the work of Manahan et al. [11,12] and Foulds et al. [13] and the relatively recent work of Catherine et al. [14] and Isselin et al. [15], all of which employs some form of stress analysis using an inverse predictive method for stress-strain behaviour that includes full consideration of plasticity. However, the work reported here focuses on the need for a more straightforward approach that can yield results at a lower cost.

\section{Method}

The experimental procedures are performed on aluminum [16] and titanium [17] metal matrix composites (MMCs) with increasing amounts of ceramic reinforcement content. The SPT specimens are initially machined into $6 \mathrm{~mm}$ diameter disc-shaped specimens with a thickness of $1 \mathrm{~mm}$. Fine grinding with Struers 4000 grit $\mathrm{SiC}$ paper and final polishing with $50 \mathrm{~nm}$ colloidal silica
(OP-S) on MD CHEM cloth further reduces the thickness to $0.5 \pm 0.005 \mathrm{~mm}$.

The SPT is conducted with a $5 \mathrm{kN}$ load cell Instron 8562 universal mechanical testing machine. The SPT is performed at ambient temperature with a speed of $0.2 \mathrm{~mm} / \mathrm{min}$. The SPT specimen is firmly clamped in place. As can be seen in Fig. 1, the SPT jig incorporates a spherical punch diameter of $2 \mathrm{~mm}$ with a lower die bore diameter of $4 \mathrm{~mm}$ and a lower die chamber edge $r^{*}$ of $0.22 \mathrm{~mm}$. The spherical ball punch is made of cast iron with a hardness of 55 HRC.

\section{Results}

In this article, a new small punch equivalent contact radius $r^{\prime}$ is proposed based on geometric analysis of a sphere and experimental small punch load displacement curve as expressed in Eq 5. The equation below takes into account the small punch elastic-plastic displacement $\delta_{y}$, which describes the evolution of the contact radius as the punch is displaced. Furthermore, $r^{\prime}$ represents the theoretical maximum contact radius that can be achieved by the SPT using Pythagoras on the triangle in Fig. 2(b).

$$
r^{\prime}=\sqrt{2 r_{o} \delta_{y}-\delta_{y}^{2}}
$$

Combining Eqs 4 and 5 leads to Eq 6, which describes the small punch maximum bend strength $\sigma_{y}$, described further in Fig. 2 .

$$
\sigma_{y}=\frac{3 P_{y}(1+\nu)}{2 \pi t_{0}^{2}} \ln \frac{R_{1}}{\sqrt{2 r_{o} \delta_{y}-\delta_{y}^{2}}}
$$

\section{Discussion}

The SPT can be thought of as a multi-mechanical test with applications in creep, stress, and fracture toughness analysis. An advantage is that samples are small enough that sampling can potentially occur in a manner that preserves the structure as a whole. In this research the SPT is utilised for assessing yield strength from small disc-shaped specimens. A correlation of the tensile yield strength $\sigma_{\mathrm{YS}}$ and the small punch maximum bend strength $\sigma_{y}$ is shown in Table 2 and Fig. 3. STE690 steel [19], 12Cr-1Mo steel [20], 6061 aluminum [21], and Zircaloy-4 [22] were analysed based on previously published work; all the other

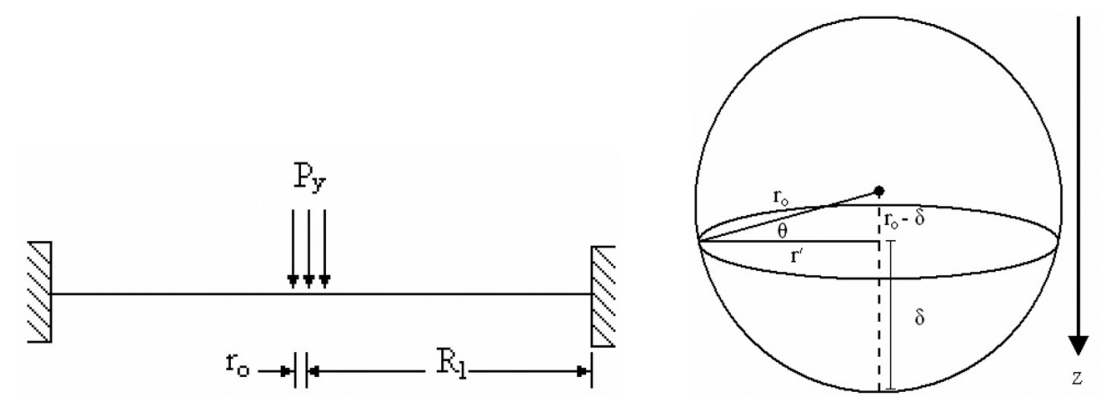

FIG. 2-(a) A flat circular plate with constant thickness [18]. (b) A proposed geometric method for determining the small punch equivalent contact radius $r^{\prime}$, where $P_{y}$ is the small punch elastic-plastic load, $R_{l}$ is the radius of the lower die bore, $r_{o}$ is the punch radius, $r^{\prime}$ is the equivalent contact radius, and $\delta$ is the small punch displacement. 
TABLE 2-Tabulated $0.2 \%$ offset proof stress and small punch maximum bend strength $\sigma_{y}$. At least three tests were conducted for each material.

\begin{tabular}{lccc}
\hline Materials & $\begin{array}{c}0.2 \% \text { Offset } \\
\text { Proof Stress, } \\
(\mathrm{MPa})\end{array}$ & $\begin{array}{c}\text { Small Punch } \\
\text { Maximum Bend } \\
\text { Strength } \sigma_{y},(\mathrm{MPa})\end{array}$ & $\begin{array}{c}\text { Percent } \\
\text { Difference, } \\
(\%)\end{array}$ \\
\hline 7A04-T6 base alloy & 462 & 442 & 5 \\
7A04/SiC/7.5p-T6 & 555 & 502 & 1 \\
7A04/SiC/10p-T6 & 557 & 576 & -3 \\
TC4 base alloy & 900 & 976 & -8 \\
TC4/TiB, TiC/2.5p, 2.5w & 909 & 1041 & -12 \\
TC4/TiB, TiC/5p, 5w & 1023 & 1088 & -6 \\
\hline
\end{tabular}

materials were tested in this study. The small punch maximum bend strength $\sigma_{y}$ was determined using Eq 6. A clear linear relationship is observed between the tensile yield strength $\sigma_{\mathrm{YS}}$ and the small punch maximum bend strength $\sigma_{y}$ over a large stress range from around $300 \mathrm{MPa}$ to $1000 \mathrm{MPa}$.
The point of non-linearity is applied in order to determine the small punch elastic-plastic load $P_{y}$. It identifies the yield load at the point of non-linearity as shown in Fig. 4. It is determined by drawing a straight line through zero and rotating the line until it is tangent to, and does not cross, the curve.

It is determined that yielding occurs early on in the deformation process of the SPT. Therefore, the small punch elastic-plastic load $P_{y}$ must be identified at the point of non-linearity. The associated small punch elastic-plastic displacements $\delta_{y}$ are identified at values less than $0.05 \mathrm{~mm}$. Therefore, careful analysis of the small punch elastic-plastic load $P_{y}$ and small punch elastic-plastic displacements $\delta_{y}$ is needed. Isselin and Shoji disputed the use of the small punch elastic-plastic load $P_{y}$, arguing that the complex biaxial stresses cannot be interpreted by analytical techniques alone [23]; however, in their research they conceded that the offset method provides a straightforward approach for identifying the small punch elastic-plastic load $P_{y}$ with reasonably good results. In addition, similar to this research, small punch displacements $\delta$

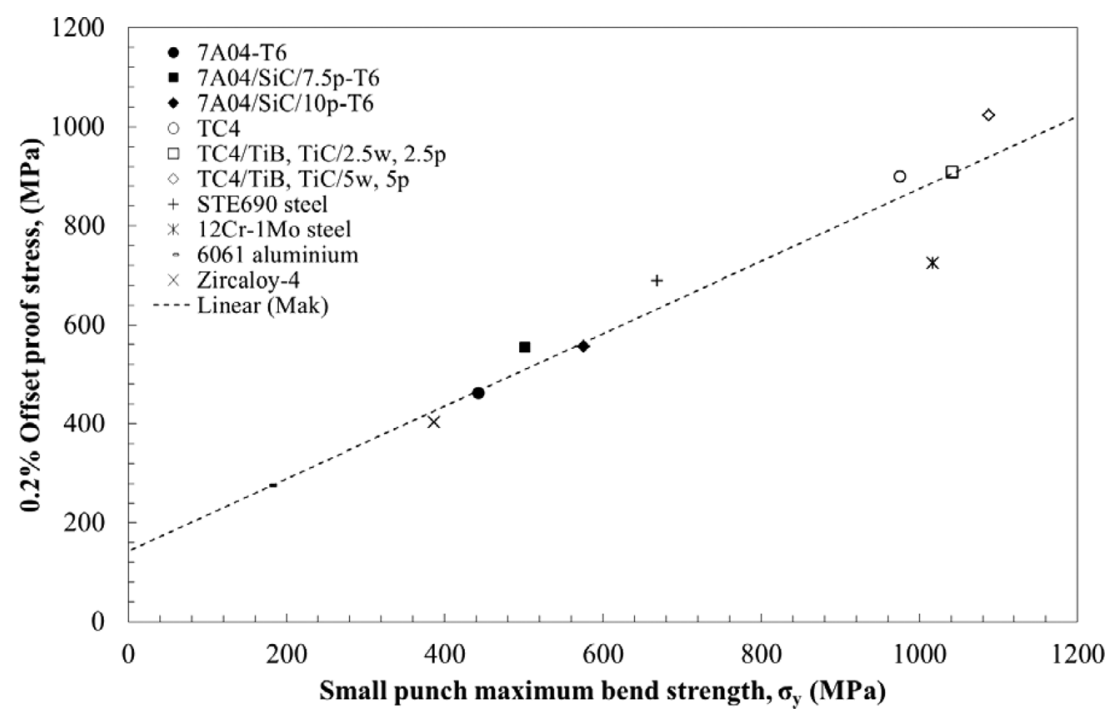

FIG. 3-A graph showing the tensile yield strength $\sigma_{Y S}$ plotted against the small punch maximum bend strength $\sigma_{y}$.

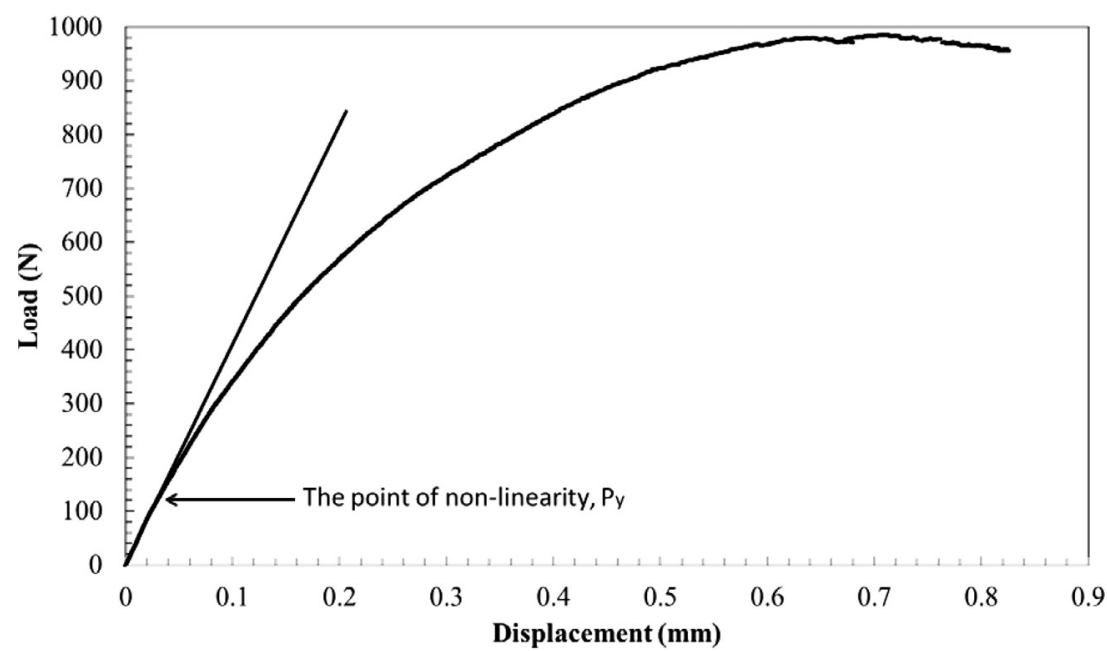

FIG. 4-Representative plot of the small punch test curve for Ti-6Al-4V showing the "point of non-linearity" method employed for identifying the small punch elastic-plastic load $P_{y}$. 
are as well identified at small displacements, which for them are below $0.02 \mathrm{~mm}$. It is true that plastic deformation occurs early on with respect to the punch and specimen; however, it is these authors' opinion that the deviation from linearity is a consequence of yielding at the free surface and not through the thickness of the specimen.

\section{Conclusions}

A new analytical solution for determining the yield strength $\sigma_{\mathrm{YS}}$ from the SPT is proposed that provides a convenient and simple method for determining tensile strength properties from alloys and MMCs. The method is based on classical plate theory of circular plates and incorporates a new analytical solution for the small punch equivalent contact radius $r^{\prime}$. The small punch equivalent contact radius $r^{\prime}$ is based on geometric analysis of a sphere and an experimental small punch load displacement curve. In particular, the small punch elastic-plastic load $P_{y}$ and corresponding small punch elastic-plastic displacement $\delta_{y}$ values are utilised. This study furthers the development of the SPT as a multi-mechanical test to be employed on all kinds of alloys and advanced MMCs. To this end, more research is required in order to standardise the test method and procedures, which will further improve the mechanical evaluation of material properties.

\section{Acknowledgments}

The writers of this work acknowledge the support of the International Science Linkage (ISL) Program (Project Nos. CG110069 and CH080230) of the Department of Innovation, Industry, Science and Research.

\section{References}

[1] Mao, X. and Takahashi, H., "Development of a FurtherMiniaturized Specimen of $3 \mathrm{~mm}$ Diameter for TEM Disk (ø $3 \mathrm{~mm}$ ) Small Punch Tests," J. Nucl. Mater., Vol. 150(1), 1987, pp. 42-52.

[2] Husain, A., Sehgal, D. K., and Pandey, R. K., "Review of Small Punch Test Technique for Evaluating Mechanical Behaviour of Materials and In-service Components Using Miniature Specimen,” J. Struct. Eng., Vol. 31(2), 2004, pp. 91-100.

[3] Xu, Y. and Zhao, Z., "A Modified Miniature Disk Test for Determining Material Mechanical Properties," J. Test. Eval., Vol. 23(4), 1995, pp. 300-306.

[4] Cheon, J. S. and Kim, I. S., "Initial Deformation during Small Punch Testing," J. Test. Eval., Vol. 24(4), 1996, pp. 255-262.

[5] Finarelli, D., Roedig, M., and Carsughi, F., "Small Punch Tests on Austenitic and Martensitic Steels Irradiated in a Spallation Environment With $530 \mathrm{MeV}$ Protons," J. Nucl. Mater., Vol. 328(2-3), 2004, pp. 146-150.

[6] Eskner, M. and Sandström, R., "Mechanical Property Evaluation Using the Small Punch Test," J. Test. Eval., Vol. 32(4), 2004, pp. 1-8.

[7] Westergaard, H. M., "Stresses in Concrete Pavements Computed by Theoretical Analysis," Public Roads, Vol. 7(2), 1926, pp. 25-35.
[8] Fleury, E. and Ha, J. S., "Small Punch Tests to Estimate the Mechanical Properties of Steels for Steam Power Plant: I. Mechanical Strength," Int. J. Pressure Vessels Piping, Vol. 75(9), 1998, pp. 699-706.

[9] Shetty, D. K., Rosenfield, A. R., Duckworth, W. H., and Held, P. R., "A Biaxial-Flexure Test for Evaluating Ceramic Strengths," J. Am. Ceram. Soc., Vol. 66(1), 1983, pp. 36- 42.

[10] Cicero, S., Lacalle, R., and Gutiérrez-Solana, F., "Application of Small Punch Techniques for the Determination of Gold Mechanical Properties," Strain, Vol. 47, 2009, pp. 484-492.

[11] Manahan, M. P., Argon, A. S., and Harling, O. K., “The Development of a Miniaturized Disk Bend Test for the Determination of Postirradiation Mechanical Properties," J. Nucl. Mater., Vol. 104, 1981, pp. 1545-1550.

[12] Manahan, M. P., Browning, A. E., Argon, A. S., and Harling, O. K., Miniaturized Disk Bend Test Technique Development and Application, ASTM STP 888, ASTM International, West Conshohocken, PA, 1986, pp. 17-49.

[13] Foulds, J. R., Woytowitz, P. J., Parnell, T. K., and Jewett, C. W., "Fracture Toughness by Small Punch Test," J. Test. Eval., Vol. 23(1), 1995, pp. 3-10.

[14] Catherine, C. S., Messier, J., Poussad, C., Rosinski, S. T., and Foulds, J. R., EPRI-CEA Finite Element Simulation Benchmark and Inverse Method for the Estimation of Elastic Plastic Behavior, ASTM STP 1418, ASTM International, West Conshohocken, PA, 2002, pp. 350-371.

[15] Isselin, J., Iost, A., Golek, J., Najjar, D., and Bigerelle, M., "Assessment of the Constitutive Law by Inverse Methodology: Small Punch Test and Hardness," J. Nucl. Mater., Vol. 352(1-3), 2006, pp. 97-106.

[16] Mak, J., Wuhrer, R., Humphries, S., Booth, N., Heness, G., Yeung, W. Y., Wei, T., Qin, J., Lu, W., and Zhang, D., "Small Punch Test of LC4/SiCp Metal Matrix Composites," Adv. Mater. Res., Vols. 123-125, 2010, pp. 439-442.

[17] Mak, J., Wuhrer, R., Heness, G., Yeung, W. Y., Callaghan, M., Wei, T., Qin, J., Lu, W., and Zhang, D., "Small Punch Test of Advanced In-Situ Synthesized Ti Metal Matrix Composites," Adv. Mater. Res., Vol. 47-50, 2008, pp. 738-741.

[18] Young, W. C., Roarke, R. J., and Budynas, R. G., Roark's Formulas for Stress and Strain, 7th ed., McGraw-Hill, New York, 2002.

[19] Abendroth, M. and Kuna, M., "Determination of Deformation and Failure Properties of Ductile Materials by Means of the Small Punch Test and Neural Networks," Comput. Mater. Sci., Vol. 28, 2003, pp. 633-644.

[20] Mao, X., Takahashi, H., and Kodaira, T., "Supersmall Punch Test to Estimate Fracture Toughness JIc and Its Application to Radiation Embrittlement of 2.25Cr-1Mo Steel," Mater. Sci. Eng., A, Vol. 150(2), 1992, pp. 231-236.

[21] Wei, T., Carr, D. G., Budzakoska, E., Payten, W., Harrison, R. P., and Ripley, M. I., "Assessment of the Fracture Toughness of 6061 Aluminium by the Small Punch Test and Finite Element Analysis," Mater. Forum, Vol. 30, 2006, pp. 39-44.

[22] Callaghan, M. D., Yeung, W. Y., Ripley, M. I., and Carr, D. G., "An Analysis of Deformation and Fracture Behaviour of Zircaloy-4 Alloy Using Small Punch Test," Mater. Sci. Forum, Vols. 475-479, 2005, pp. 1415-1420.

[23] Isselin, J. and Shoji, T., "Yield Strength Evaluation by Small-Punch Test," J. Test. Eval., Vol. 37(6), 2009, pp. 1-7. 